\title{
Melt Undercooling and Nucleation Kinetics
}

\author{
J.H. Perepezko ${ }^{1, a}$, and G. Wilde ${ }^{2, b}$ \\ ${ }^{1}$ University of Wisconsin-Madison, Department of Materials Science and Engineering, 1509 \\ University Ave., Madison, WI 53706, USA \\ ${ }^{2}$ University of Münster, Institute of Materials Physics, Wilhelm-Klemm-Str. 10, 48149 Münster,
}

Germany

\author{
aperepezk@engr.wisc.edu \\ bwilde@uni-muenster.de
}

\begin{abstract}
While melt undercooling is often observed during solidification the control of undercooling and nucleation is difficult due to the numerous possible heterogeneous sites present in even high purity melts. The identification of active nucleation sites has been a continuing challenge that requires developing well planned experimentation. In samples with well-defined and controlled undercooling, the identification can be established for a number of the active sites and mechanisms that can act to catalyze nucleation. The sites and mechanisms that have been identified involve primary phases developed during cooling of alloy melts, liquid-added particle interfaces being modified (e.g. by adsorption or reaction) creating a particle type independent nucleation potency, dissolved impurities precipitating out of the melt at high undercooling or acting to alter local atomic arrangements in the melt to catalyze nucleation and nucleation sites

resulting from residual solid preserved in cavities in inclusions or surface coatings. Recent advances in clarifying the structure of liquids reveal local atomic arrangements such as short range order, medium range order and cluster structures, but the role of these local heterogeneities on the undercooling and crystallization behavior is incompletely understood at present.
\end{abstract}

\section{Introduction}

Liquid undercooling plays a central role in determining the solidification microstructure [1,2]. Even though the study of undercooling phenomena has a long history dating from the first report by Fahrenheit [3 ], undercooling behavior continues to attract increasing attention as new experimental methods, characterization techniques and modeling approaches have clarified the understanding of the liquid atomic structure and new applications such as nanomaterials [4] and phase change devices [5] have emerged that rely on undercooling and nucleation for their synthesis and functional operation. However, achieving reliable and reproducible control over 
the observed undercooling level has been a continuing challenge. Since only a single heterogeneous nucleation event is sufficient to initiate solidification in an undercooled melt and since there are numerous potential nucleation sites in an undercooled bulk melt, the identification of the active nucleant has been a difficult task with only limited success. For nucleant activity it is useful to consider two regimes. For example, in grain refining, the objective is to minimize the undercooling in order to allow the maximum number of grain refining particles to initiate grain formation [6] that is covered in the review by StJohn. Other examples also indicate that at low undercooling solidification is initiated at pre-existing crystal sites [7]. At the opposite extreme, metastable and amorphous phases can be synthesized at high undercooling by rapid quenching [8] or by special melt treatments such as fluxing or containerless processing $[9,10]$.

Another approach to achieve high undercooling is to employ the droplet sample method [11]. Upon subdivision of a melt into a large population of fine droplets a finite number of nucleants can be isolated into a few droplets to allow the nucleant-free droplets to exhibit large undercooling. In addition, the development of bulk metallic glasses has generated a renewed interest in the use of flux treatment of the melt where the flux acts to remove nucleants or to deactivate them to allow for large undercooling [12-14]. Associated with the experience on undercooling behavior there are many reports concerning the influence of melt superheat and thermal cycling, but a satisfactory explanation has not been available to account for these effects until recently. However, systematic studies of thermal cycling effects have provided new insight into the operation of a nucleant refining mechanism that promotes enhanced undercooling during flux treatment.[14] Moreover, advances in high rate calorimetry have provided a basis for a rational explanation for the effect of melt superheat on the subsequent undercooling [7 ]. In spite of the uncertainties regarding the action of specific nucleants, it is possible to provide insight into undercooling mechanisms and to achieve some measure of control through a systematic analysis of the crystallization kinetics behavior in undercooled melts and amorphous phases [14-20]. In fact, the activity has been so extensive that within the available coverage it is not possible to discuss all aspects satisfactorily. Instead the focus of the discussion is on undercooling, heterogeneous nucleation mechanisms and some aspects of nucleation analysis.

\section{Nucleation Catalysis by Primary Phases}

Under most conditions, solidification is initiated by a heterogeneous nucleation event. While the study of homogeneous nucleation requires a sample free from all external nucleants, the study of heterogeneous nucleation requires a sample which contains only well-known and characterized nucleants. A proper examination of nucleation catalysis requires the full identification of the catalytic sites and solidification product structure as well as a characterization of the potency of the catalytic site in terms of the interaction energetics (i.e. contact angle) between the nucleus and catalyst. To circumvent the difficulties encountered in bulk systems, a droplet technique involving droplets on an "inert" substrate, droplet emulsions or entrained droplets has often been utilized to examine nucleation catalysis [11, 21-25]. 
Many studies have been conducted on binary alloys in order to determine the catalytic effect of crystals of a primary solid solution phase on the nucleation of the liquid where it has been demonstrated [26] that each characteristic contact angle between the nucleant and the liquid will yield a discrete, well-defined undercooling on nucleation which can be used to describe catalytic potency. However, for a given system different undercooling values have been observed in different investigations. Part of the cause for the disagreement between the observations of previous studies may be attributed to spurious effects introduced by the use of different types of substrates or materials to envelop the liquid metal $[11,27]$. For example, in the fabrication of samples for the entrained droplet method, the liquid droplets represent the final portion of the sample to solidify and as a result are the portions most likely to contain segregated concentrations of residual impurities. To permit a resolution of these differences, the droplet emulsion technique (DET) $[11,27]$ was adapted to study primary phase catalysis in simple eutectic or peritectic alloy systems. The strategy of the approach is shown in Figure 1. By using alloy emulsions that have been proven to exhibit a high undercooling before nucleation and equilibrating alloys in the liquid $+\alpha$ or liquid $+\beta$ two-phase field, the catalytic effect of $\alpha$ or $\beta$ on subsequent nucleation of the liquid can be examined without the possible interference from a foreign substrate [28]. An important preliminary step in the application of the DET to the study of heterogeneous nucleation is the evaluation of the possible catalytic potency of the droplet surface coating and other possible sites. This was accomplished by determining the maximum undercooling $\Delta \mathrm{T}_{\mathrm{e}}$ below the eutectic to nucleation of a droplet emulsion which was initially completely liquid. Specially designed thermal cycles can be employed to demonstrate that the intended nucleation site is indeed the one that is operating [11]. This is not possible with other methods, but is of crucial importance in obtaining valid heterogeneous nucleation temperatures.

\section{Nucleation Catalysis by Incorporated Particles}

In melts with incorporated particles such as in the solidification processing of particle reinforced composites most of the attention has been focused on the interaction between the particles and the solidification front. However, it is recognized that the particles can also act to catalyze nucleation. In bulk melts it is not possible to identify the catalytic action of individual particles separately even though substantial undercooling can be observed in melts with dispersed particles [29], but the identification can be accomplished with droplet samples and specific treatments $[30,31]$.

In order to conduct experiments on added nucleants in a melt, it is first necessary to remove or isolate background nucleants. Breaking the sample into droplets achieves isolation of background nucleants to reveal the characteristic undercooling due to the added particles by containing them in only a portion of the droplets. Droplets without a potent background nucleant are able to reach large undercoolings or in the case where nucleants under investigation are added to the melt, reveal the added nucleant potency. As shown in Figure 2a, a melt containing background nucleants and added particles forms small droplets when emulsified. Any given droplet may contain both a background nucleant and an added particle, either a background 
nucleant or an added particle, or neither. All droplets however will have a coating that is often an oxide that may serve as a nucleation site. Figure $2 b$ shows a schematic of four possible nucleation sites; 1) a background nucleant, 2) the droplet coating, 3) a compound site involving both the added particle and the droplet coating, and 4) the added particle.

In order to demonstrate the effectiveness of this experimental design, Sn droplets were examined with differential thermal analysis (DTA) to identify nucleation sites and their characteristic undercooling and potency [30]. Figure 2c shows DTA curves of the Sn droplets in three conditions; (a) pure Sn droplets, (b) Sn droplets with added $\mathrm{Y}_{2} \mathrm{O}_{3}$ particles, and (c) Sn droplets with added $\mathrm{Y}_{2} \mathrm{O}_{3}$ particles that have undergone a surface modification treatment. As can be seen in curve (a) the pure $\mathrm{Sn}$ droplets have one peak at a nucleation temperature of $56^{\circ} \mathrm{C}$. With the added $\mathrm{Y}_{2} \mathrm{O}_{3}$ particles, a second peak at a nucleation temperature of $96^{\circ} \mathrm{C}$ develops as shown in curve (b). This second peak illustrates the characteristic nucleation potency of the $\mathrm{Y}_{2} \mathrm{O}_{3}$ particles. The remaining peak at $56^{\circ} \mathrm{C}$ indicates that not all of the droplets contain a $\mathrm{Y}_{2} \mathrm{O}_{3}$ particle such that the particle free droplets still nucleate on the same nucleation sites as in the pure Sn sample. Sn droplets that have added $\mathrm{Y}_{2} \mathrm{O}_{3}$ particles and have undergone a surface modification treatment are shown in curve (c). As illustrated in the curve, the peak at $56^{\circ} \mathrm{C}$ that was the characteristic nucleation peak in the pure $\mathrm{Sn}$ droplets shifts to $79^{\circ} \mathrm{C}$ with the surface modification. This indicates that the nucleation site is the surface coating (depicted as site 2 in Figure $2 \mathrm{~b}$ ). The peak at $96^{\circ} \mathrm{C}$ that was present in curve (b) remains. This indicates that the $\mathrm{Y}_{2} \mathrm{O}_{3}$ particles are acting alone and not in conjunction with the surface coating.

Figure 3a shows the solidification behavior for Sn droplets with different added particle catalysts. Surprisingly all the oxide particles exhibit the same characteristic undercooling. The interfacial energies of the solid Sn, liquid Sn and particle are not the same for all the particles as would be necessary for the same $f(\theta)$ value and the corresponding characteristic undercooling. The mechanism for this behavior is shown schematically in Figure 4a. An adsorbed layer of $\mathrm{Sn}$ is shown on the particle catalysts. It is this layer that catalyzes Sn solidification [31]. If all the oxide particles have a surface layer of oxygen atoms, a similar adsorbed patch on each of the oxide particles with the same catalytic potency can form. This phenomenon was not only observed with oxide particles. Similar Sn droplets made with added telluride and sulfide particles also exhibited consistent catalytic potency regardless of specific particle type. One possible explanation for the consistency in catalytic potency of different oxide particles is the reduction of the particles by the Sn. Examination of the thermodynamics of such reactions reveals that this explanation is not viable. All oxide particles shown in Figure 3a have positive free energies of reaction when considering reduction by liquid $\mathrm{Sn}$ [32].

Another mechanism for the operation of incorporated particle catalysis is demonstrated in Figure $3 b$. In this case both $\mathrm{CdO}$ and $\mathrm{PbO}$ particles exhibit the same nucleation undercooling, but the undercooling level is distinctly different than that for other oxide particles shown in Figure 3a. However, the free energy change for the reduction of both $\mathrm{CdO}$ and $\mathrm{PbO}$ in $\mathrm{Sn}$ is favorable [32] 
In this case, it appears that the incorporated particles undergo a reaction with the $\mathrm{Sn}$ melt to change their original identity. The resulting reaction layer then acts as the nucleant site for Sn as illustrated in Figure 4b [31]. This is similar to the adsorption model for heterogeneous nucleation [25].

From the early work of Turnbull and Vonnegut [33] and Bramfitt [34] lattice misfit has been used extensively to attempt to rationalize the observed undercooling behavior during heterogeneous nucleation. However, from the results above and other work [35] it is evident that the observed undercooling and nucleation behavior deviates from a lattice misfit criterion at high undercooling. Moreover, there is clear evidence that a solid in contact with a liquid can influence the local atomic arrangements in the liquid $[36,37]$. The observed liquid undercooling may be lowered or enhanced depending on the nature of the interaction [37, 38]. Consequently, while there may be some merit to view lattice misfit and interface matching during the heteroepitaxy that can occur at low undercooling, at high undercooling chemical interaction effects appear to be the dominant factor in controlling the undercooling response.

\section{Environment Effects on Nucleation Undercooling}

There is a wealth of experience on the processing of metallic melts to control impurity levels and remove inclusions. The relevance of this experience to melt undercooling behavior is evident, but only a few studies have been reported to establish this link. Moreover, since liquids are usually processed in a crucible in a gaseous atmosphere, environmental interaction can be expected to affect the observed undercooling.

Fluxing treatments, like background nucleant isolation, have proven successful in removal of background nucleants and to elucidate nucleation behavior [12-14, 39-43]. As an example, a 100 mg sample of super purity gold (Alfa, 99.9999\%) was coated with Pyrex glass and examined with DTA. Multiple melting/freezing cycles of the same $100 \mathrm{mg}$ sample were conducted. Figure 5a shows the undercooling in the gold sample as a function of cycle number. As can be seen in the figure, the initial undercooling is relatively small. As the sample is cycled under an argon atmosphere the undercooling values get progressively larger until a steady state undercooling of $212^{\circ} \mathrm{C}$ is reached after approximately 20 cycles. The microstructure of the gold sample after solidifying from high undercooling is only one dendritic grain. Figure 5a also shows the effects of adding oxygen to the Ar purge gas. As seen in the figure, undercooling drops significantly with the addition of 500 parts per million (ppm) oxygen. When the purge gas is returned to pure Ar, the high degree of undercooling is restored after $\sim 20$ cycles, similar to the behavior observed during the initial 20 cycles of the experiment.

The 20 cycle transient behavior in the repeated cycling test can be explained by a nucleant refining mechanism where impurities that form precipitating phases are purged from the sample [14]. Oxide forming impurities along with the dissolved oxygen in gold can precipitate out solid phase clusters that can act as nucleants. The impurities are dissolved in the liquid gold at high 
temperatures, however as the gold is cooled the impurities saturate and eventually form oxide precipitates. Once precipitated the oxide can nucleate solid gold. Subsequent oxides that form are then pushed by the gold dendrite to the sample surface or entrapped in the dendrite. Oxides that are pushed to the surface are then passed into the flux, removing them from the sample. On the next melting/freezing cycle the sample has partially purged the impurity; for gold the impurity is likely iron [14]. Impurity saturation and oxide formation then requires a lower temperature to occur thus resulting in a higher undercooling for solidification as noted in Figure 5b. The impurity purging process progresses until the steady state undercooling is achieved. The development of a saturation or steady-state undercooling with this process indicates equilibrium between the sample and the DTA chamber environment that can be controlled if the concentration of either the impurity or oxygen is fixed by some external factor (e.g., the oxygen content in the DTA atmosphere). When oxygen is added to the Ar purge gas, the oxygen dissolved in the sample is replenished resulting in a decreased undercooling. When the oxygen is subsequently removed from the Ar purge gas, the impurity purging mechanism resumes and the undercooling returns to the steady state undercooling.

A steady-state undercooling level could also occur if the nucleant removal is sufficiently effective so that another nucleant becomes active as observed for flux treated $\mathrm{Cu}$ [43]. However, as noted by Turnbull [44] it is difficult to imagine how a solid nucleant dispersed within the melt or at the Pyrex-gold interface could endure the numerous thermal cycles in an unaltered condition. In fact, when applying the model by Pound and La Mer [45], which treats nucleation on an impurity cluster that is smaller than the size of the critical nucleus, for the case of $\mathrm{Au}$ of high purity, a maximum size of the impurity cluster of less than 10 atoms has been determined [46]. Certainly it is difficult to envision such a small cluster to survive several hundred melting cycles without losing its structural integrity due to entropy-driven dissolution.

A similar process has been identified for the modification of Al-Si alloys by $\mathrm{P}$ in entrained droplet studies [47-49]. In this case the nucleation of Si is catalyzed by the formation of AlP precipitates at the liquid-solid interface. The observed undercooling is correlated with the residual $\mathrm{P}$ content even at the few ppm level. A thermodynamic analysis provided a quantitative confirmation of the strong sensitivity of the undercooling to the P content [50].

In other related work, Cramb [51-53] has carried out a series of well-designed, systematic studies on the effect of environmental conditions on the undercooling and wetting behavior of $\mathrm{Fe}$ and $\mathrm{Ni}$ on various crystalline oxide substrates. It should be noted that in most previous work the undercooling measurements were conducted on samples in a "clean" environment, but the actual oxygen pressure, $\mathrm{Po}_{2}$ was not reported and no observations were made on the wetting behavior between the sample and the substrate. In the studies reported by Cramb and coworkers there was a direct relationship between the observed undercooling and the $\mathrm{Po}_{2}$. Similar to the behavior observed for gold, the undercooling decreased with increasing $\mathrm{Po}_{2}$ for values of $\mathrm{Po}_{2}$ above the decomposition value for the oxide and there was a concurrent decrease in the wetting angle. The 
undercooling values observed were substantial (i.e. greater than $100 \mathrm{~K}$ ) and there was no correlation between the undercooling for different substrates and the lattice misfit between Fe or $\mathrm{Ni}$ and the substrates [51].

Other work related to the effect of impurities on the undercooling of alloys with glass forming compositions has documented the significant influence of minor impurity levels in limiting the amount of liquid undercooling. In this case impurities at a low level (i.e. microalloying) can act either to enhance or to diminish the glass forming ability (GFA) of an alloy. For example, in $\mathrm{Zr}$ base alloys (Figure 6) residual oxygen at the few ppm level significantly degrade the GFA [54, 55], but this effect can be alleviated partially by minor additions of B and C [56, 57]. Similarly, the minor addition of $\mathrm{Y}$ to Fe based glass forming alloys enhances the GFA presumably due to the high affinity of Y for residual oxygen [58]. It has also been reported that oxygen levels above 1 at.. \% in a Zr-base amorphous alloy result in an increase in liquid viscosity [59].

Another role of impurities has been proposed in recent work by Rappaz [60]. In Au alloys it appears that a long standing industrial method of grain refinement is to add minor amounts of Ir. In the grain refined $\mathrm{Au}$ alloys the grains exhibit a much enhanced twin formation compared to unrefined alloys. From an elegant analysis, it was suggested that the Ir addition promoted the development of icosahedral local atom arrangements that acted as nucleation sites. A similar behavior was also observed in Al-Zn alloys following the minor addition of $\mathrm{Cr}$ [61]. In a study of the undercooling behavior of nanosize Ga drops, minor additions of Si were observed to promote the crystallization of metastable phases and it was suggested that structural motifs other than icosahedral may be present in undercooled liquids that can act to catalyze metastable structures $[62,63]$. Similarly, in glass forming Pd alloys structural investigation of flux treated samples revealed a densification of the undercooled liquid that was manifested in an increased resistance to crystallization compared to samples without flux treatment [64]. Other structural studies of a Zr-base amorphous alloy revealed a change in short range order above the glass transition [65].

\section{Nucleation Analysis}

A review of the atomistic modeling of solidification is covered in the contribution from Karma and Tourett and only selected developments are treated here. An important advance in analysis that is receiving continued attention is the establishment of the nucleation theorem [66-69]. The nucleation theorem is derived from the free energy change, $\Delta \mathrm{G}$ or work for the formation of a cluster of size $\mathrm{n}$ at a chemical potential difference (i.e. supersaturation or undercooling) $\Delta \mu$ as

$$
\Delta G(n, \Delta \mu)=-n \Delta \mu+\Phi(n, \Delta \mu)
$$

where $\Phi(n, \Delta \mu)$ is the cluster excess free energy (i.e. cluster surface energy). The nucleation theorem is then expressed by 


$$
\frac{d \Delta G^{*}}{d \Delta \mu}=-n^{*}+\frac{d \Phi\left(n^{*}, \Delta \mu\right)}{d \Delta \mu}
$$

where the starred quantities are evaluated at the critical nucleus size. Since in many cases $\Phi$ changes only negligibly with $\Delta \mu$, the second term in equation 2 vanishes and the nucleation theorem is

$$
\frac{d \Delta G^{*}}{d \Delta \mu}=-n^{*}
$$

In a more detailed analysis $\mathrm{n} *$ is replaced by $\Delta \mathrm{n} *$ which is the excess number of atoms over that present in the same volume of original phase. The significance and value of the nucleation theorem is based upon thermodynamics and makes no assumptions about the critical nucleus size and shape so that it is valid at the smallest scale. While the results often involve excess quantities they are independent of the dividing interface. There are several forms of the nucleation theorem that apply to different nucleation conditions (i.e. isothermal, isobaric, continuous cooling). The expression can be related to experiment by expressing the nucleation rate, $\mathrm{J}$ in general as

$$
J=A \exp \left(\frac{-\Delta G^{*}}{k T}\right)
$$

Where $\mathrm{A}$ is a prefactor with a form that depends on the specific nucleation process $(70,71)$. Rearranging equation 4 gives

$$
\Delta G^{*}=-k T \ln J+k T \ln A
$$

Then

$$
\Delta n^{*}=\frac{d(k T \ln J)}{d \Delta \mu}-\frac{d(k T \ln A)}{d \Delta \mu}
$$

The application of the different forms of the nucleation theorem has been substantiated for vapor to liquid reactions. For undercooled melts $\mathrm{J}$ has a very strong temperature dependence compared to the prefactor A so that over a narrow temperature range the second term in equation 6 can be neglected. However, over a more extended temperature range that is important in rapid solidification or in the crystallization of metallic glass where the prefactor A is strongly temperature dependent and $\Delta \mu$ is related to the undercooling, accurate knowledge of $\mathrm{A}$ is necessary. A further discussion of the nucleation theorem is presented in two excellent comprehensive treatments on nucleation which also cover other current developments $(70,71)$.

There have also been important developments in the experimental and computational analysis of nucleation kinetics. In specific cases that are governed by high initial purity and continuous purification through fluxing as well as by comparatively large solubility for (metallic) impurities, 
as in the case of most (liquid) transition metals, conditions can be obtained so that the kinetics can be described by an analysis based on homogeneous nucleation although the absence of very small impurity clusters due to the unavoidable presence of minute amounts of impurities cannot be proven. In a series of experiments on transition metals with fcc equilibrium crystal structure, quantitative nucleation rate measurements have been conducted that were based on the stochastic nature of the nucleation process $[44,72,73]$. Similar results and conclusions have been obtained for pure $\mathrm{Zr}$ that has been melted and undercooled within an electrostatic levitation facility [74]. For the case of pure $\mathrm{Ni}$, the experimental analysis was compared to Monte Carlo simulations in conjunction with umbrella sampling and parallel tempering [75] using an embedded atom method (EAM) potential proposed by Foiles [76] to model the interactions between the Ni atoms. Experimentally, repeated undercooling measurements have been performed on a single bulk sample under well-defined and identical conditions. Building on the analysis developed by Perepezko et al. [77] that allows direct extraction of nucleation rates from undercooling data without any approximations and without relying on a specific nucleation model, statistically significant nucleation undercooling data were obtained and analyzed as cumulative probability distributions. Additionally, in order to extend the range of nucleation rates that are accessible to experimental analysis, the cooling rates as well as the sample volume have been varied systematically.

The nucleation rate $\mathrm{J}(\Delta \mathrm{T})$, as obtained from the experiments on the different Ni samples, is displayed in Figure 7, spanning eight orders of magnitude. A continuous temperature dependence is seen, indicating that only one type of nucleation site is active throughout the measurements. Surface nucleation can be discarded as an active mechanism since the data of $\mathrm{J}$ for different sample masses seem to follow a smooth curve without discontinuities between the data sets that correspond to different masses. For this it is crucial that the samples are encased in glass, providing an isolation of the sample from the crystalline container walls and an enhancement of the purity of the samples through oxide dissolution [14]. In contrast, methodologies that rely on applying non-catalytic coatings do not work at the high temperatures that have to be considered in materials such as $\mathrm{Ni}$ [71]; in particular, because the surfaces of high melting metals are prone to developing active nucleation sites due to their high reactivity.

In addition to the sample masses, also the purity of the Ni samples has been varied from $99.999 \%$ to $99.6 \%$. As can be inferred from Fig. 7, the purity variation has no visible influence on the nucleation rate. This seems to indicate that the experiments were not affected by nucleation on impurities. One has to keep in mind, however, that the purity variation does not specifically target any potential nucleation sites and thus, the obtained values of $\mathrm{J}$ might be affected by a constant impurity of low potency. While for the cluster sizes that are relevant for nucleation at high undercooling even melts of the highest purity will contain impurities, this does not mean that the impurities in solution will necessarily catalyze nucleation. Moreover, the results obtained on materials from different sources and with different nominal impurity level show no distinction concerning their nucleation behavior, which strongly supports the view that 
solidification in the present cases was initiated by homogeneous nucleation. In a recent study, a similar experimental approach has been applied for a binary $\mathrm{Cu}-\mathrm{Co}$ alloy that presents a metastable miscibility gap in the undercooled liquid state (as in fact many glass-forming compositions do) [78]. In that case, the topology of the miscibility gap serves to introduce an additional, non-linear dependency of the driving force for nucleation on undercooling. Yet, due to the embedding of the liquid metal inside another liquid metal of different composition, encountering homogeneous nucleation conditions is favored [78].

From the experimentally determined nucleation rates, the height of the activation barrier for nucleation, $\Delta \mathrm{G}^{*}$, was calculated and compared to $\Delta \mathrm{G}^{*}$ obtained from the Monte Carlo simulations. The results shown in Figure 7 indicate excellent agreement between the completely independent data sets. Figure $7 \mathrm{~b}$ shows that the simulation results for $\Delta \mathrm{G}^{*}$ are well described by classical nucleation theory $(\mathrm{CNT})$. Also, the data point for the highest undercooling $\Delta \mathrm{T}=448 \mathrm{~K}$ agrees well with the fitted dashed line according to CNT. At latter value of $\Delta \mathrm{T}$, the critical cluster size is roughly $\mathrm{n}^{*} \approx 200$ corresponding to a critical barrier of $\Delta \mathrm{G}^{*} \approx 2.3 \mathrm{eV}$. The simulation results also indicated that the curvature corrections to the interfacial free energy are already relatively small for clusters containing of the order of $n=200$ atoms. Thus, the evidence supports the application of CNT kinetics in this case. This is also obvious with respect to the experimental data. The range of $\Delta \mathrm{G}^{*}$ in the experiments is roughly between $5.5 \mathrm{eV}$ and $8.5 \mathrm{eV}$. According to the simulations, the critical nuclei contain between about 500 to 1000 atoms in that range of barriers. Thus, for undercooling regimes that are accessible to controlled melt-cooling experiments, classical nucleation theory provides a good description of the effective kinetics.

\section{Effects of melt superheat on nucleation}

The degree of superheating of a melt prior to its subsequent undercooling and resulting crystallization has been demonstrated to play a significant role in determining the solidification behavior, in particular, for the nucleation process and the accessible undercooling [79-82]. For example, for a Zr-based amorphous alloy systematic experiments have demonstrated that oxygen plays a key role in the heterogeneous nucleation through the action of oxide particles [83, 84] in a manner similar to nucleant refining [14]. Yet, our understanding of the relationship between prior overheating and undercooling is still rather incomplete. Most of the prior studies examined bulk samples of the melt where extraneous nucleants of different kind can control the response of the system to undercooling. Hence, the relationship between undercooling and melt superheat has not been specified or explained in a sufficiently comprehensive form. With the development of nanotechnology and microelectromechanical systems (MEMS), it is possible to fabricate calorimeter sensors that are able to measure samples with nanogram masses and energies less than one nanojoule or even picojoule [85-88]. These developments enable the possibility of considerably enlarging and controlling the scanning rates.

Single pure Sn droplets were analyzed with differential fast scanning calorimetry (DFSC) to investigate the effects of melt superheat and cooling rate on nucleation undercooling [7]. The DFSC technique allows for cooling rates from 500 to $10,000 \mathrm{~K} / \mathrm{s}$ to be investigated. Superheating 
up to $92 \mathrm{~K}$ above the observed melting temperature was also investigated. As shown in Figure 8a the observed undercoolings in the Sn droplet are a function of cooling rate and superheat. As can be seen in figure $8 \mathrm{a}$, when the superheating is greater than $26 \mathrm{~K}$, the observed undercooling increases slightly with increased cooling rate from $\sim 95 \mathrm{~K}$ at $500 \mathrm{~K} / \mathrm{s}$ to $98 \mathrm{~K}$ at $10,000 \mathrm{~K} / \mathrm{s}$. This behavior is due to the stochastic nature of nucleation and the higher cooling rates reducing the time available for a nucleation event to occur. For superheats of $26 \mathrm{~K}$ and below there is a transition cooling rate above which higher cooling rates result in a reduction in the observed undercooling.

The reduction in undercooling with increased cooling rates can be explained using a modification of the heterogeneous nucleation model on cavity preserved solid developed by Turnbull [89]. At a modest superheat residual solid Sn can be preserved in cavities in the oxide coating on the droplet. The larger the cavity diameter the faster the residual solid will melt on heating and regrow on cooling [90]. Thus a smaller diameter cavity will contain residual solid Sn at higher superheating or longer holding times above the melting temperature than a larger cavity. This is shown schematically in Figure 8b. For large superheats (>26 K) the residual solid is completely melted and nucleation occurs without the catalysis of cavity preserved solid. For the modest superheats the time that the sample is at a temperature above the melting temperature is important. As can be seen in Figure 8a, cooling slowly from a $26 \mathrm{~K}$ superheat results in an undercooling similar to the samples exposed to higher superheating. Thus the time the sample was above the melting temperature was sufficient to completely melt the residual solid $\mathrm{Sn}$ in even the smallest cavities. However, when the cooling rate is increased above $5000 \mathrm{~K} / \mathrm{s}$ residual Sn survives and serves as a nucleation site. Increasing the cooling rate to $10,000 \mathrm{~K} / \mathrm{s}$ preserves more residual $\mathrm{Sn}$ in larger cavities further reducing the undercooling. Similar trends are observed at superheats below $26 \mathrm{~K}$ with lower transition cooling rates. It should be noted that when the superheating was less than $13 \mathrm{~K}$, the sample solidified with minimal undercooling.

\section{Finite-size effects}

The enhancement of undercooling by sample size reduction is due to two effects related to the melt volume. The major effect is due to nucleant isolation. For a given nucleant concentration, $\mathrm{m}$ the probability that a given volume will retain a nucleant that terminates the undercooling by crystallization is either $\exp (-\mathrm{ma})$ or $\exp (-\mathrm{mv})$ depending on whether the nucleant is surface area,a or volume, $v$ distributed. The second more minor effect is related to the stochastic nature of nucleation where the nucleation rate, $\mathrm{J}$ represents the probability of nucleation in a unit time on an area or volume site. Since $J$ is related to $\exp \left(-1 /\left(T \Delta T^{2}\right)\right)$, the undercooling scales with either $\ln (\mathrm{a})$ or $\ln (\mathrm{v})$.

There is another significant effect of sample size reduction which is related to the effect of changing thermodynamics upon reaching the nanoscale size range. With the reduction of the size of a thermodynamic system, interface-related terms of the excess thermodynamic potentials become significant or even dominant for nanoscale systems, thus giving rise to size-dependent 
phase equilibria. Since the transition rates of first-order phase transformations depend sensitively on the thermodynamic potentials of the parent - and the new phase, with their difference defining the so-called "driving force" of the transformation, also the kinetics of phase transformations are affected by finite size effects. It should be emphasized, that such finite size effects need to be considered even for system sizes of several tens of nanometers, i.e. at a size scale where the competition between the size of the entire system and the size of the nucleus of the new phase can safely be neglected. From the thermodynamics of confined systems, much work has been devoted to the size dependence of the melting temperature of single-component materials, elucidating the impact of the external interface (the surface) on the thermodynamics of nanoscale systems. Much less attention has been devoted onto the more complex case of multi-component (and multiphase) nanoscale materials, where in addition to the external interfaces excess contributions to the thermodynamic potentials also arise due to the excess energy associated with internal heterophase interfaces which is dependent on temperature- or composition-dependent variations of the relative amount of matter per phase. While it is established that interfacial enrichment or depletion in solute (interfacial segregation [91]) and elastic interactions between the interfaces and the bulk (interface stress [92]) can significantly affect the relative stability of single-phase states in nanoscale alloys at constant interfacial area, the consequences of capillarity for the two-phase coexistence within a particle remain widely unexplored. Yet, the capillary energy of the interface between coexisting phases can lead to significant changes in the constitutional phase diagram, i.e. of the composition-temperature fields in which the different phases represent the thermodynamically stable state, and which may be observable even for sizes as large as $100 \mathrm{~nm}$, i.e. well above the structure size of next-generation microelectronics devices [93]. These changes are not mere shifts of temperatures or of compositions at equilibrium; instead, several qualitative rules, which are universally obeyed in conventional alloy phase diagrams of macroscopic systems, are no longer applicable at the nanometer scale. This has been demonstrated for the case of a binary nanoparticle, which, in bulk, would present a simple eutectic phase diagram with negligible terminal solubilities in the solid state. Taking explicitly the contributions of the interface excess energy density of heterophase interfaces for all twophase states into account leads to deviations from the Gibbs approach to constructing phase diagrams, where interface contributions are mostly neglected. While Gibbs was aware of that fact, and rightly neglected it for the bulk-type of materials for which he developed the thermodynamic description, the interface excess leads to non-linear dependence between nominal composition and molar free energy of two-phase states [94]. Thus, the common tangent construction is no longer valid. The resulting phase diagrams for the bulk system and for two alloy particles with different sizes are summarized in Figure 9. It is seen that, as the particle size is reduced, the phase diagram undergoes several qualitative changes, each of which breaks one of the rules that apply universally to the construction of the phase diagram for macroscopic systems. First, it is observed that the invariance of the solidus temperature is lost in favor of a significant composition-dependence. Second, as illustrated by the colored lines representing states of identical composition $\mathrm{x}_{\mathrm{L}}$ of the liquid phase at equilibrium, the compositions of the 
constituent phases in two-phase equilibria are no longer invariant at constant temperature.

Thirdly, the equi-composition lines lose their continuity at the intersection with the liquidus line. This implies that there is a discrete jump in liquid fraction across the liquidus of the small alloy particles, consistent with the result of numerical modeling matched to $\mathrm{Sn}-\mathrm{Bi}$ nanoparticles [95], where the ends of the tie lines were found to detach from the phase boundary lines.

However, the most fundamental consequence of the finite system size is a topological change in the phase diagram with the degeneration of the eutectic point of the macroscopic system into a line representing an interval of compositions $\Delta \mathrm{x}_{\mathrm{d}}$ (defined in Figure 9 (c)) for which the particle undergoes a discontinuous transition between the two-phase solid-solid state and the singlephase liquid state. In the macroscopic system, three phases can coexist at equilibrium at the eutectic point; by contrast, discontinuous melting in this model is a transition between a twophase equilibrium (solid-solid) and a single-phase state, without three-phase equilibrium (clearly, three phases will coexist during melting, but this situation resembles a transient, nonequilibrium configuration). It is because of this loss of three-phase equilibrium in the finite-size system that the transition from a eutectic point to a discontinuous melting line can be reconciled with the phase rule. In fact, recent experimental studies of isothermal composition variation within the electron microscope [96] as well as calorimetric investigations on a Bi-Cd eutectic that closely resembles the assumptions of the simple model eutectic $[94,97]$ are in complete agreement with the model results. The results corroborate the importance of internal heterophase interfaces for phase equilibria and thus for predicting phase transformations and their nucleation kinetics in nanostructured materials.

\section{Summary}

The analysis of undercooling and nucleation requires careful experimental design.

Heterogeneous nucleation can occur at numerous competing sites and isolating or identifying the active nucleation sites is nontrivial. As illustrated in this review, seemingly pure materials still contain uncontrolled elements that can serve to catalyze nucleation. Even when controlled substances are added to a melt, the interaction of the melt and the added substance (e.g. adsorption or reaction) can result in nucleation behavior that is difficult to anticipate. Particularly with nucleation of new phases in nanoscale systems, the complex and intricate interplay of thermodynamic and kinetic finite-size effects needs to be taken into account. However, meaningful conclusions and understanding can be achieved with clean experiments and analysis models. The elucidation of the multiple effects of impurities is clearly an important area for further development, but the identification of the complete mechanism for impurity influences on undercooling is a challenging task. For the most part in the analysis of solidification behavior the attention has been focused on the evolving solid phase and the liquid phase has been treated as structurally homogeneous with smoothly varying composition gradients. However, there is now compelling evidence from a number of different structural measurements that undercooled liquids are spatially heterogeneous and exhibit a variety of local atomic arrangements that are a 
function of alloy composition and very sensitive to minor impurities. The details of liquid structure are important and must be considered in the overall analysis.

\section{Acknowledgement}

The past support for JHP from the US Army Research Office for studies on solidification of undercooled melts and the National Science Foundation for studies of solidification catalysis as well as the current support (NSF DMR 1332851) is gratefully acknowledged. Portions of this work developed during a sabbatical visit by JHP to the University of Münster that was supported by the Alexander von Humboldt Foundation. GW gratefully acknowledges support by the Alexander von Humboldt Foundation and by the Deutsche Forschungsgemeinschaft.

\section{References}

[1] M.C. Flemings: Solidification Processing, McGraw-Hill, 1974.

[2] W. Kurz and D.J. Fisher: Fundamentals of Solidification, 3rd ed., Trans Tech, 1992

[3] D.B. Fahrenheit, Phil. Trans. Royal Soc. of London 39, 78 (1724).

[4] E.J. Schwalbach and P.W. Voorhees, Nano Lett., 8, 3739 (2008).

[5] J. Orava, A.L. Greer, B. Gholipour, D.W. Hewak and C. E. Smith, Nature Mater. 11, 279 (2012).

[6] J.H. Perepezko: in ASM Handbook Volume 15: Casting 276-287 (ASM International, 2008). J. H. Perepezko in Metals Handbook, Ninth Edition, volume 15 - Casting, p. 101 (1988).

[7] B. Yang, J H. Perepezko, J. W. P. Schmelzer, Y. Gao, and C.Schick, J. Chem. Phys. 140, 10451 (2014).

[8] W.J. Boettinger, J.H. Perepezko: In: Liebermann HH, editor. Rapidly solidified alloys: processes, structures, properties, applications. New York (NY): Marcel Dekker; p. 17 (1993).

[9] D.M. Herlach, Ann. Rev. Mat. Sci., 21, 23 (1991).

[10] D. M. Herlach, Metals, 4, 196 (2014).

[11] J. H. Perepezko, Mater. Sci. Eng., 65, 125 (1984).

[12] H.W. Kui, A.L. Greer, and D. Turnbull, Appl Phys Lett. 45, 615 (1984).

[13] A. Inoue and N. Nishiyama, Mater Sci Eng. A226-A228, 401 (1997).

[14] G. Wilde, J.L. Sebright and J.H. Perepezko, Acta Materialia, 54, 4759 (2006).

[15] J. Bokeloh, R.E. Rozas, J. Horbach, G. Wilde, Phys Rev Lett, 107, 145701 (2011).

[16] J. Bokeloh, G. Wilde, JOM, 66, 1512 (2014).

[17] S. Klein, D. Holland-Moritz, D.M. Herlach, Phys. Rev. B, 80, 212202 (2009).

[18] J.H. Perepezko, C. Santhaweesuk, J.Q. Wang and S.D. Imhoff: Jnl. Alloys Comp.. (2013). doi:10.1016/j.jallcom.2013.11.220

[19] D. Turnbull, Acta Metall., l. 8 (1953).

[20] D. Turnbull, J. Chem. Phys., 198 (1950). 
[21] B. E. Sundquist and L. F. Mondolfo, Metall. Trans. A1ME, 221, 157 (1961).

[22] R. T. Southin and B. A. Chadwick, Acta Metall., 20, 223 (1978).

[23 K. I. Moore, D. L. Zhang and B. Cantor, Acta Metall. Mater.,38, 327 (1990).

[24]W.T. Kim and B. Cantor, J. Mater. Sci., 26, 2868 (1991).

[25] W.T. Kim and B. Cantor, Acta Metall. Mater., 42, 3115 (1994).

[26] M. E. Glicksman and W. J. Childs, Acta Metall., 10, 925 (1962).

[27] I. E. Anderson and J. H. Perepezko: in G. J. Abbaschian and

S. A. David (eds.), Grain Refinement in Castings and Welds, Metallu.rgical Society of AIME, Warrendale, PA, p.67 (1983).

[28] J.H. Perepezko and M.J. Uttormark, Metall Mater Trans A., 27A, 269 (1996).

[29] G. Wilde and J.H. Perepezko, Mater. Sci. Eng. A, A283, 25 (2000).

[30] M.K. Hoffmeyer and J. H. Perepezko, Scr. Metall., 22, 1143 (1988).

[31] J.H. Perepezko, M.K. Hoffmeyer and M.P. DeCicco, Metall and Mater. Trans. (in press).

[32] O. Kubaschewski, C.B. Alcock and P.J. Spencer, Materials Thermochemistry, 6th

Edition,Pergamon Press, Oxford, (1993).

[33] D. Turnbull and R. Vonnegut, Ind. Eng. Chem., 44, 1292 (1952).

[34] B.L. Bramfitt, Metall. Trans., 1, 1987 (1970).

[35] P. J. Shambergera and M. J. O’Malley, Acta Mater. 84, 265 (2015).

[36] S.H. Oh, Y. Kaufmann, C. Scheu, W.D. Kaplan and M. Rühle, Science, 310, 661 (2005)

[37] T.U. Schülli, R. Daudin, G. Renaud, A. Vaysset, O. Geaymond and A. Pasturel, Nature, 464, 1174 (2010)

[38]A.L. Greer, Nature Mater. 5, 13 (2006).

[39] T.Z.Kattamis and M.C. Flemings, Trans AIME, 236, 1523 (1966).

[40] G. Wilde, G.P. Görler, R. Willnecker and G. Dietz, Appl Phys Lett, 65, 397 (1994).

[41] D. Granata, E.Fischer, V. Wessels and J.F. Löffler, Acta Mater. 71, 145 (2014).

[42] D. Granata, E.Fischer, V. Wessels and J.F. Löffler, Appl. Phys. Lett., 106, 011902 (2015)

[43] G. Wilde, C. Santhaweesuk, J.L. Sebright, J. Bokeloh, J.H. Perepezko, J. Phys. Cond. Mat., 21, 464113 (2009).

[44] D. Turnbull, Prog Mater Sci, 269 (1981).

[45] G.M. Pound, V.K. La Mer, J. Am. Chem. Soc., 74, 2323 (1952).

[46] J. Bokeloh, G. Wilde, R.E. Rozas, R. Benjamin, J. Horbach, Eur. Phys. J. Special Topics, 223, 511 (2014).

[47] M. Zarif, B. Mckay and P. Schumacher, Metall. Mater. Trans., 42A, 1684 (2011).

[48] J.H. Li, M. Albu, F. Hofer and P. Schumacher, Acta Mater., 83, 187 (2015).

[49] J.H. Li, M.Z. Zarif, M. Albu, B. Mckay, F. Hofer and P. Schumacher, Acta Mater., 72, 80 (2014).

[50] S-M. Liang and R. Schmid-Fetzer, Acta Mater., 72, 41 (2014).

[51] S. Ueda, H. Shi, X. Hang, H. Shibata and A.W. Cramb, Metall Mater Trans B, 34B, 503 (2003).

[52] M. E. Valdez, P. Uranga, K. Fuchigami, H. Shibata and A.W. Cramb, Metall Mater Trans B, 37B, 811 (2006). 
[53] M.E. Valdez, P. Uranga and A.W. Cramb, Metall Mater Trans B, 38B, 257 (2007).

[54] X.H. Lin, W.L. Johnson and W.K. Rhim, Materials Trans. JIM, 38, 473-477 (1997).

[55] W.L. Johnson, MRS Bull. 42(1999).

[56] Y. Zhang, D.Q. Zhao, M.X. Pan and W.H. Wang, J. Non-Cryst. Solids, 315, 206 (2003).

[57] A. Inoue, T. Negishi, H. Kimura and T. Aoki, Mater. Trans. JIM, 38, 185 (1997).

[58] Z.P. Lu and C.T. Liu, J. Mater. Res. 19, 92 (2004).

[59] A. Kübler, J. Eckert, A. Gebert and L. Schultz, J. Appl. Phys.83, 3438 (1998).

[60] G. Kurtuldu, A. Sicco and M. Rappaz, Acta Mater. 70, 240 (2014).

[61] G. Kurtuldu, P. Jarry and M. Rappaz, Acta Mater., 61, 7098 (2013).

[62] E.A. Sutter, P.W. Sutter, E. Uccelli and A.F. Morral, Phy. Rev. B, 84, 193303 (2011).

[63] G.B. Parravicini, A. Stella, P. Ghigna, G. Spinolo, A. Miglion, F. d'Acapito and R. Kofman, Appl. Phys. Lett., 89, 033123 (2006).

[64] N. Chen, L. Gu, G.Q. Xie, D.V. Louzguine-Luzgin, A.R. Yavari, G. Vaughan, S.D. Imhoff, J.H. Perepezko, T. Abe and A. Inoue, Acta Mater., 58, 5886 (2010).

[65] H. Schumacher, U. Herr, D. Oelgeschlaeger, A. Traverse and K. Samwer, J. Appl. Phys., 82, 155 (1997).

[66] D. W. Oxtoby and D. Kashchiev, J. Chem. Phys. 100, 7665 (1994).

[67] D.W. Oxtoby and A. Laaksonen, J. Chem. Phys., 102, 6846 (1995).

[68] D. Kashchiev, J. Chem. Phys. 76, 5098 (1982) and 125, 014502 (2006).

[69] I.J. Ford, Phys. Rev. E, 56, 5615 (1997).

[70] D. Kashchiev, Nucleation-Basic Theory with Applications, (Butterworth-Heinemann, oxford0 (2000).

[71] K.F. Kelton, A.L. Greer, Nucleation in Condensed Matter - Applications in Materials and Biology (Elsevier, Amsterdam) (2010)

[72] J. Bokeloh, R.E. Rozas, J. Horbach, G. Wilde, Phys Rev Lett, 107, 145701 (2011).

[73] J. Bokeloh, G. Wilde, JOM, 66, 1512 (2014).

[74] S. Klein, D. Holland-Moritz, D.M. Herlach, Phys. Rev. B, 80, 212202 (2009).

[75] D. Frenkel and B. Smit, Understanding Molecular Simulation (Academic Press, San Diego, 2001).

[76] S. M. Foiles, Phys. Rev. B, 32, 3409 (1985).

[77] M. J. Uttormark, J.W. Zanter, J.H. Perepezko, J. Cryst. Growth, 177, 258 (1997).

[78] Y. Zhang, C. Simon, T. Volkmann, M. Kolbe, D.M. Herlach, G. Wilde, Appl Phys Lett, 105, 041908 (2014).

[79] P. Rudolph, H. J. Koh, N. Schäfer, and T. Fukuda, J. Cryst. Growth 166, 578 (1996).

[80] H. J. Koh, P. Rudolph, N. Schäfer, K. Umetsu, and T. Fukuda, Mater. Sci. Eng. B 34, 199 (1995).

[81] Z. Zhou, W. Wang, and L. Sun, Appl. Phys. A 71, 261 (2000).

[82] M. Mühlberg, P. Rudolph, M. Laasch, and E. Treser, J. Cryst. Growth 128, 571 (1993). 
[83] S. Mukherjee, Z. Zhou, J. Schoers, W.L. Johnson and W.K. Rhim, Appl. Phys. Lett., 84, 5010 (2004).

[84] J.J. Wall, C.T. Liu, W.K. Rhim, J. Z. Li, P.K. Liaw and W.L. Johnson, Appl. Phys. Lett., 92, 244106 (2008).

[85] S. L. Lai, J. Y. Guo, V. Petrova, G. Ramanath, and L. H. Allen, Phys. Rev. Lett. 77, 99 (1996).

[86] A. A. Minakov and C. Schick, Rev. Sci. Instrum. 78, 073902 (2007).

[87] M. Zhang, M. Y. Efremov, F. Schiettekatte, E. A. Olson, A. T. Kwan,S. L. Lai, T. Wisleder, J. E. Greene, and L. H. Allen, Phys.Rev.B62,10548 (2000).

[88] E. Zhuravlev and C. Schick, Thermochim. Acta 505, 1 (2010).

[89] D. Turnbull, J. Chem. Phys. 18, 198 (1950).

[90]J. Schmidt, J.V. Wittemann, and U. Gösele, Chem. Rev., 110, 361 (2010).

[91] J. Weissmüller, Nanostruct Mater, 3, 261 (1993.

[92] J. Weissmüller, C. Lemier, Phys Rev Lett, 82, 213 (1999).

[93] G. Wilde, Surf. Interf. Analysis (SIA), 38, 1047 (2006).

[94] J. Weissmüller, P. Bunzel, G. Wilde, Scripta Mater, 51, 813 (2004).

[95] W.A. Jesser, G.J. Shiflet, G.L. Allen, J.L. Crawford, Mater Res Innovat, 2, 211 (1999).

[96] J.G. Lee, H. Mori, Phil Mag, 84, 2675 (2004).

[97] G. Wilde, P. Bunzel, H. Rösner, J. Weissmüller, J. Alloys Comp, 434-435, 286 (2007). 


\section{Figure Captions}

Fig. 1-General thermal treatment for solidification catalysis experiments with alloy droplets. Phase diagram related to schematic thermogram (on right)

Fig. 2-Nucleant isolation (a) shown schematically during droplet emulsification, (b) four potential nucleation sites within a droplet and (c) DTA curves for Sn droplets. Melting behavior is shown in the top curve, a) shows the solidification behavior of the pure $\mathrm{Sn}$ droplets without particle addition, b) shows the solidification behavior of $\mathrm{Sn}$ droplets with added $\mathrm{Y}_{2} \mathrm{O}_{3}$ particles and c) shows the solidification behavior of surface modified Sn droplets with incorporated $\mathrm{Y}_{2} \mathrm{O}_{3}$ particles.

Fig. 3-a) DTA curves showing a consistent nucleation temperature with different oxide particle additions. b) DTA curves showing the nucleation behavior of Sn droplets containing $\mathrm{CdO}$ and $\mathrm{PbO}$ particles.

Fig. 4 a) Schematic of $\beta$-Sn nucleating on an adsorbed patch on a particle catalyst. Nucleation occurring on an adsorbed patch allows for a consistent nucleation potency and characteristic undercooling even with different types of particle catalysts. b) Illustration of potential reaction between the Sn liquid and particle substrate, forming a nucleation catalysis layer in systems with thermodynamically favorable reduction of the particle by liquid Sn.

Fig. 5 a) Observed undercooling in a super purity gold sample as a function of number of melting/freezing cycles. Inset shows the first 30 cycles and the establishment of the steady state undercooling regime. The gray areas marked "Ar+Air" were cycled under a partial pressure of air, as the Ar flow rate was reduced during these cycles. Cycles 500-700 were conducted with Ar mixed with $500 \mathrm{ppm} \mathrm{O}_{2}$ as the cover gas. All other cycles were conducted with a pure Ar cover gas. b) Schematic of nucleation refining mechanism that results in incremental undercooling increase.

Fig. 6 Time-Temperature Transformation curves illustrating the strong influence of oxygen content on the nucleation behavior (from 54 ).

Fig. 7-Temperature dependence of nucleation rates from the experiments for different system sizes, as indicated. The solid line corresponds to a fit according to classical nucleation theory.

Fig. 8- $\Delta \mathrm{G}^{*}$ as a function of $\Delta \mathrm{T} / \mathrm{T}_{\mathrm{m}}$. Results from the $\mathrm{MC}$ simulation for three different system sizes (symbols) are shown. The experimental data (bold lines) are extracted from the different samples, as indicated in Fig. 7. The dashed line is a fit to simulation data by classical nucleation theory for a box containing $\mathrm{N}=8788$ atoms.

Fig. 9-a)-Observed undercoolings in a Sn droplet as a function of superheating and cooling rate. b) Schematic of cavities in the Sn droplet's oxide coating that are capable of preserving residual solid $\mathrm{Sn}$ above the melting temperature of the bulk droplet. 
Fig. 10- (a) Phase diagram of a bulk eutectic alloy with no solid solubility. Black: phase coexistence lines; colored: lines of equal solute fraction $\mathrm{x}_{\mathrm{L}}$ in the liquid phase for three arbitrarily chosen values of $\mathrm{x}_{\mathrm{L}}$. (b) and (c): as in (a), but finite size systems with particle diameters $\mathrm{D}=50$ $\mathrm{nm}$ and $\mathrm{D}=5 \mathrm{~nm}$ respectively. $\Delta \mathrm{x}_{\mathrm{d}}$ : discontinuous melting interval where a direct transition from a two-phase solid to a single-phase liquid without three-phase coexistence occurs. Capital letters indicate the phases that are stable in the respective regions of temperature/composition space. $\mathrm{S}_{1,2}$ : solid phases; L: liquid. The gray shades represent the topologic features of the bulk phase diagram for easier comparison. 


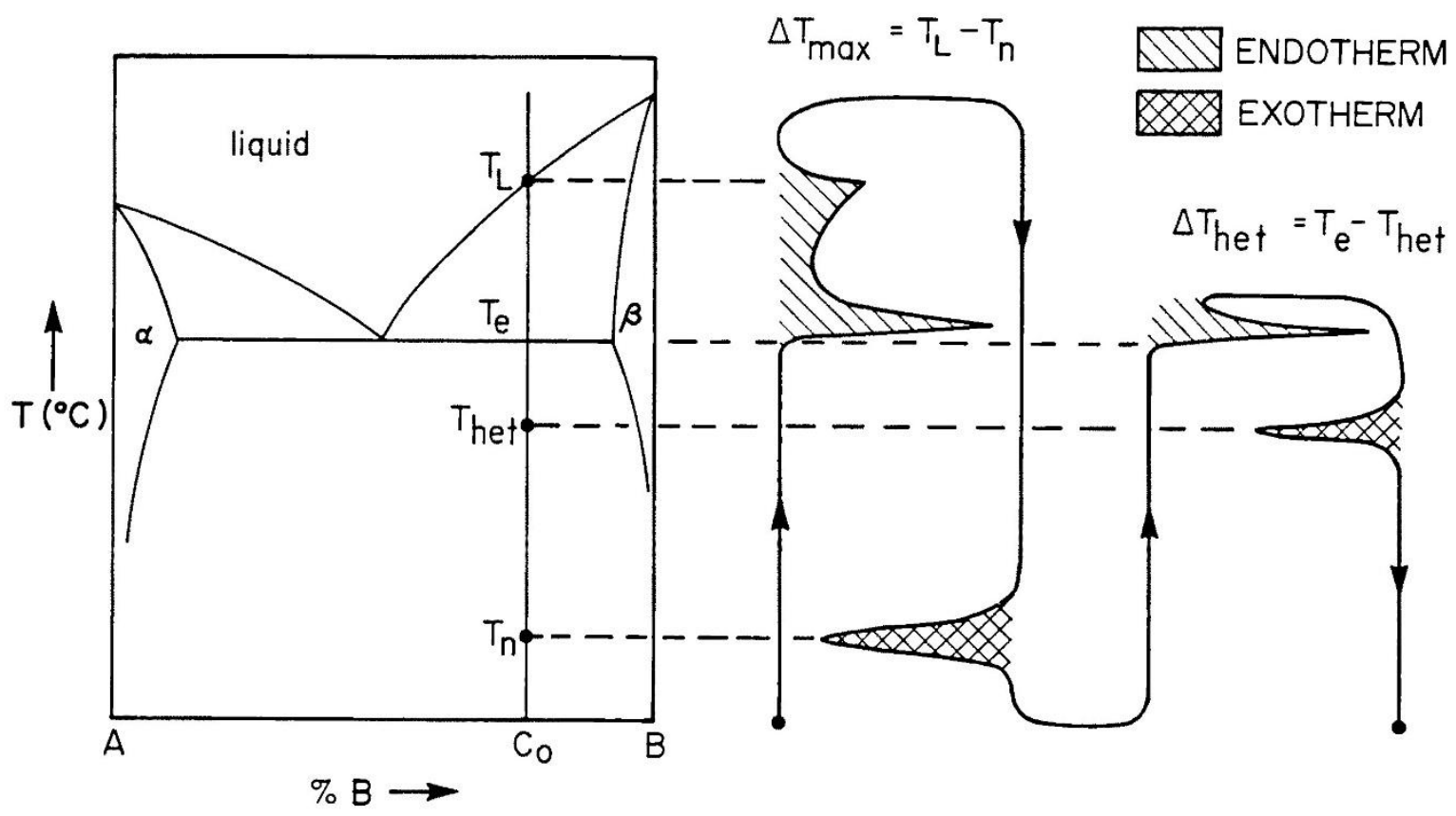

Fig. 1

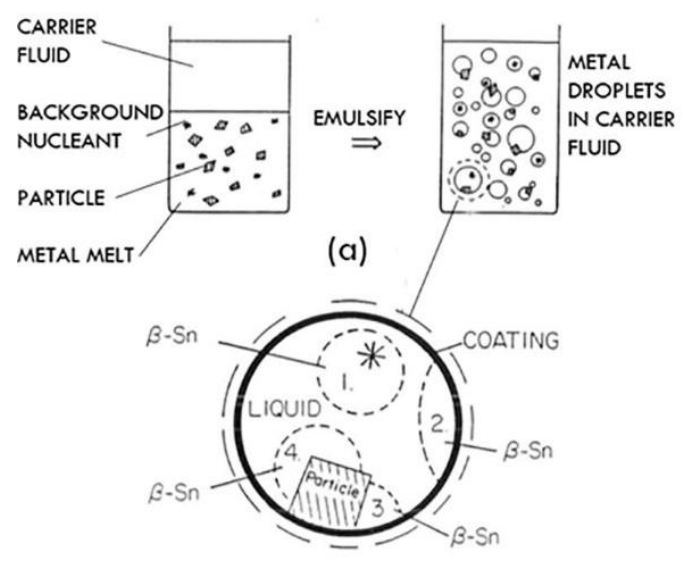

(b)

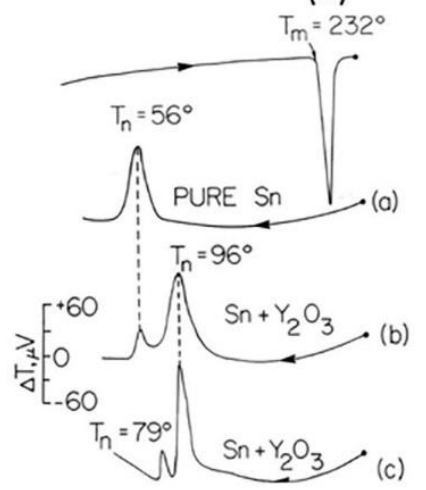

(c) 
Fig. 2

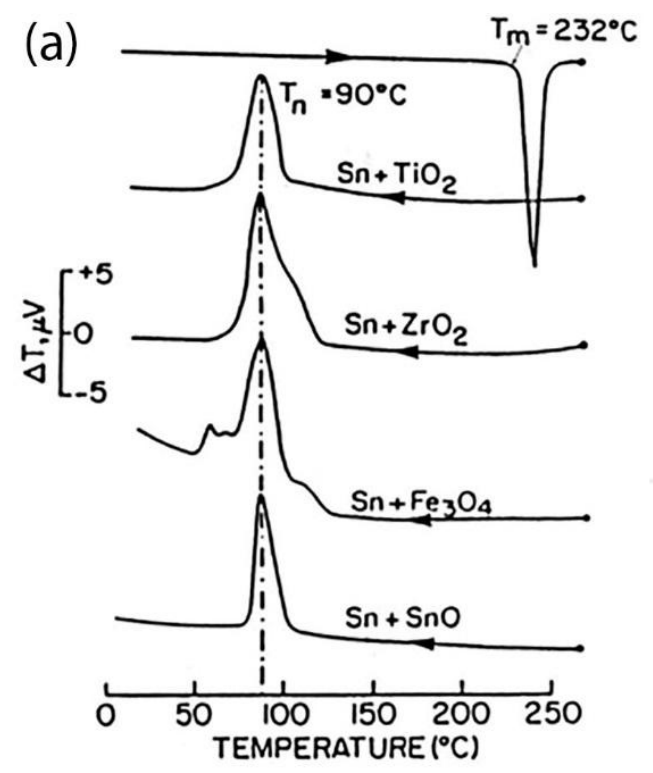

(b)

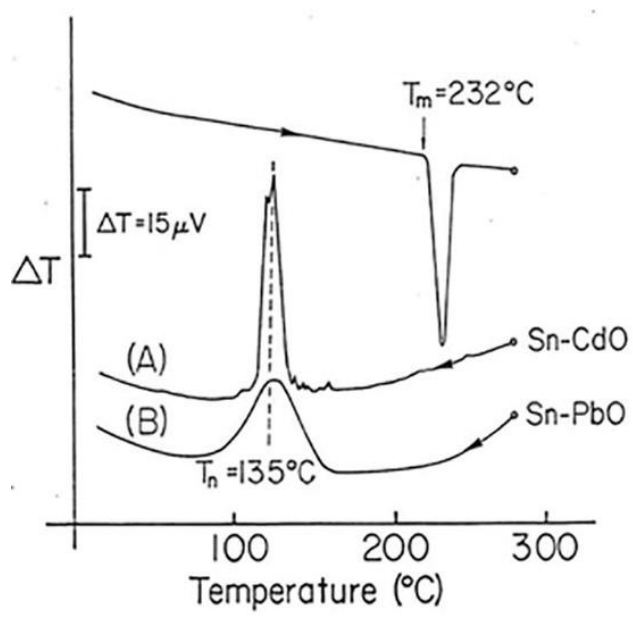


Fig. 3

(a) Liquid

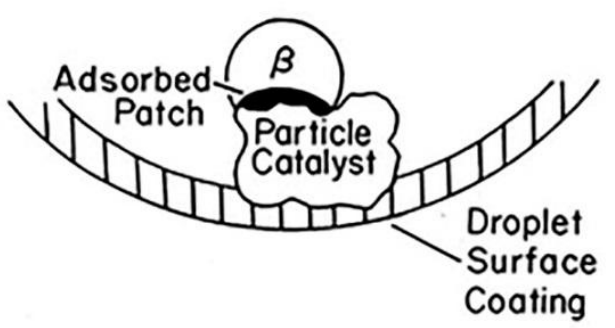

(b)

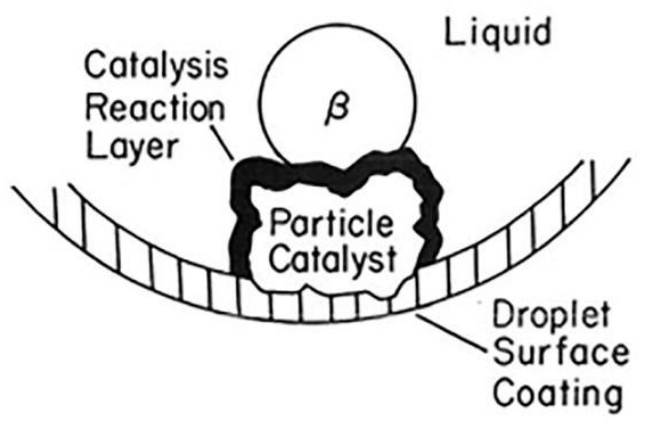


Fig. 4

(a)

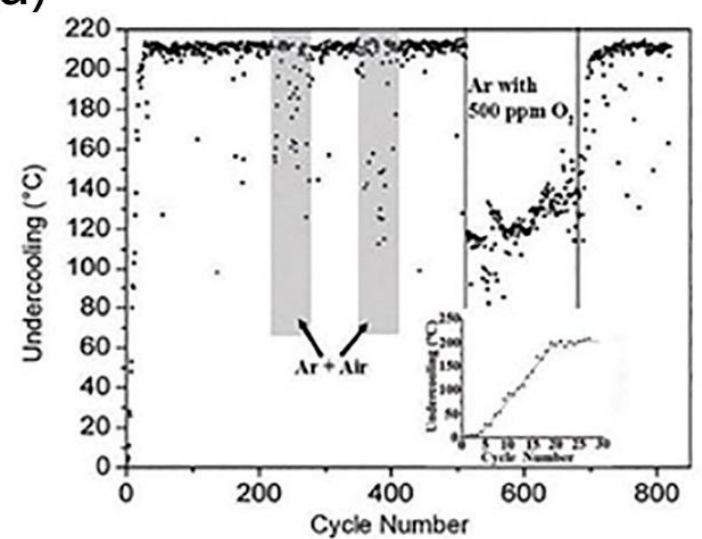

(b)

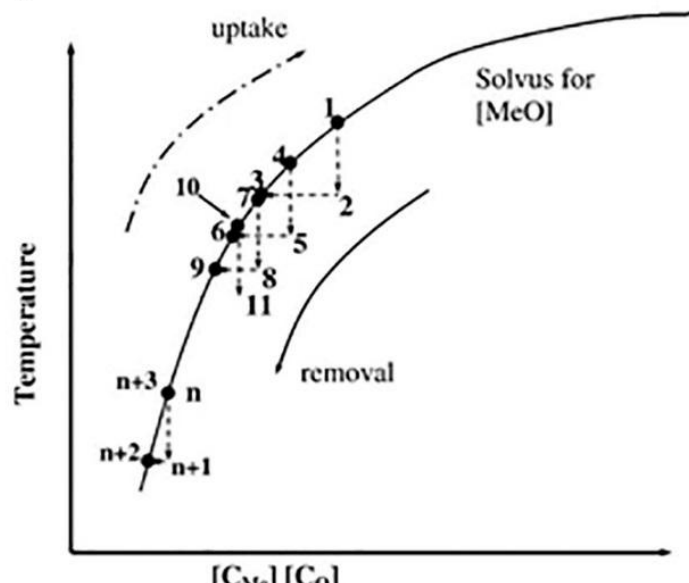

$\left[\mathrm{C}_{\mathrm{ste}}\right]\left[\mathrm{C}_{\mathbf{o}}\right]$ 
Fig. 5

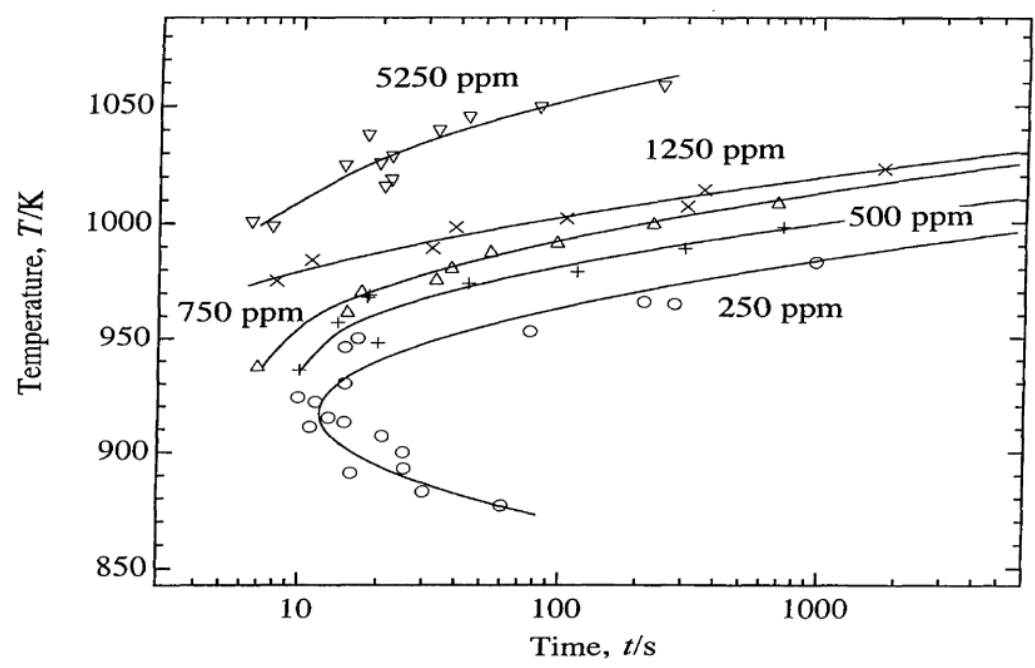

Fig. 6 


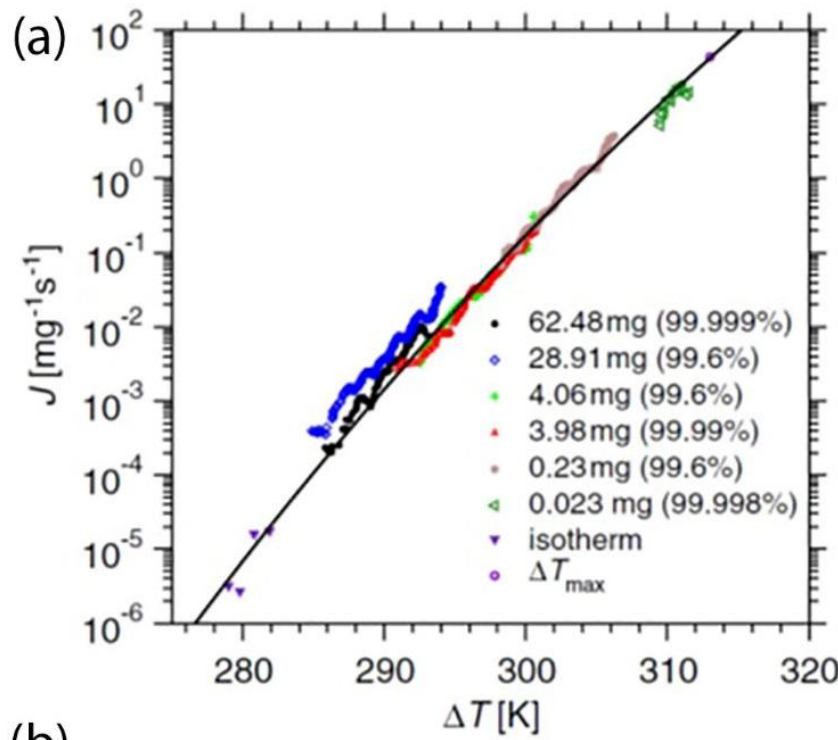

(b)

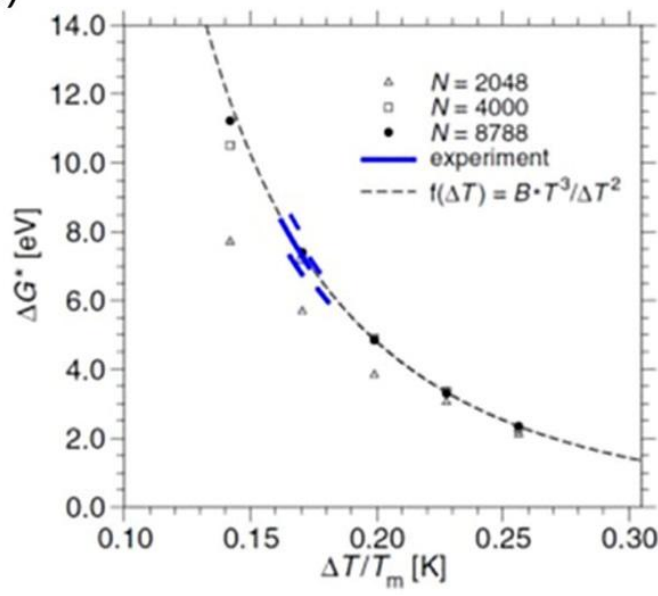


Fig. 7

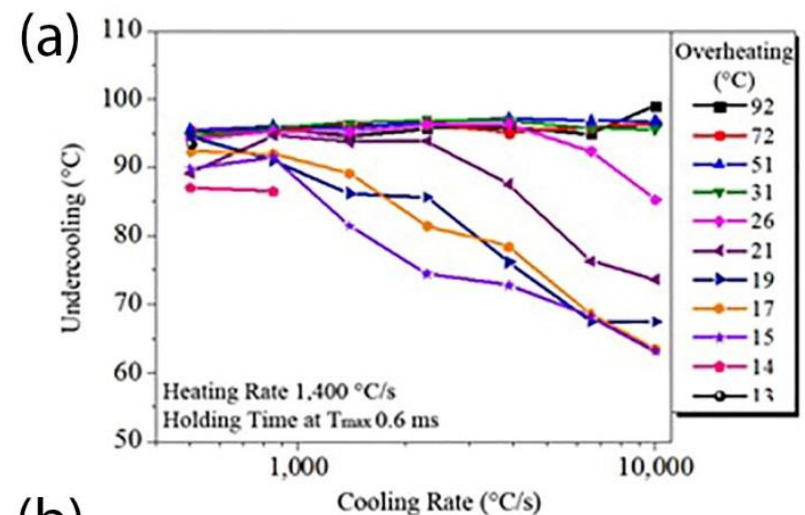

(b)

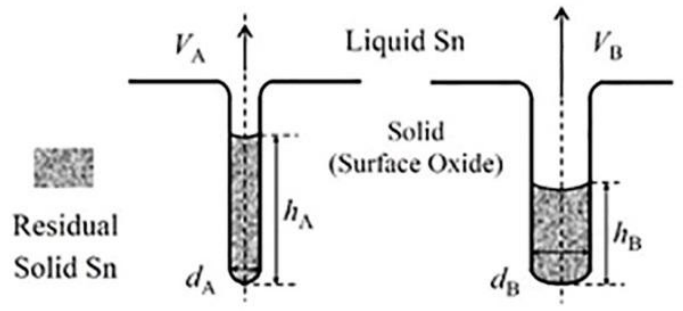

Site A Site B

Fig. 8 

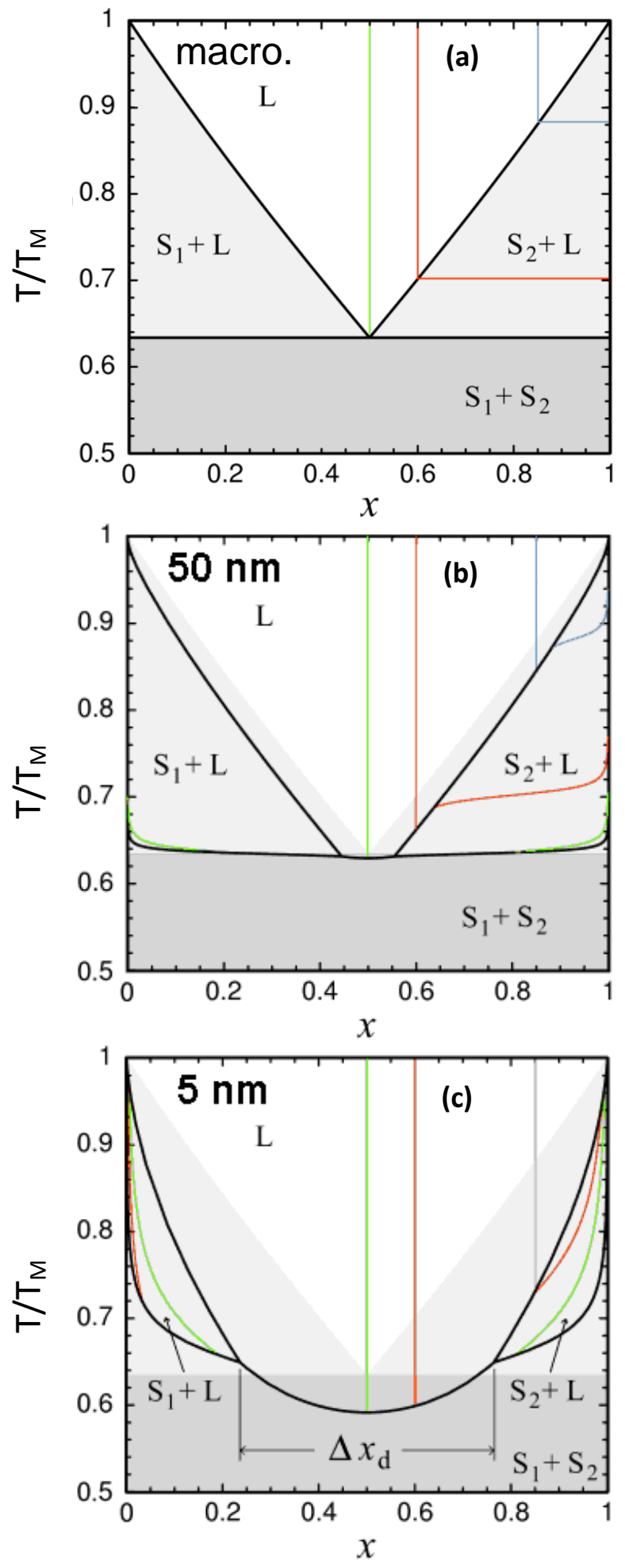

Fig. 9 